$\underline{\text { Research Article }}$

\title{
Burden of pneumococcal disease in children in Cuba before the introduction of a novel pneumococcal conjugate vaccine
}

\author{
Nivaldo Linares-Pérez ${ }^{1}$, Brian Wahl' ${ }^{2}$ Misladys Rodriguez ${ }^{3}$, Gilda Toraño ${ }^{4}$, María E. Toledo-Romaní ${ }^{3}$ \\ ${ }^{1}$ Finlay Vaccine Institute, Clinical Research and Impact Evaluation Division, Havana, Cuba, ${ }^{2}$ Johns Hopkins Bloomberg School of Public Health, \\ International Vaccine Access Center (IVAC), Department of International Health, Baltimore, MD, USA, ${ }^{3}$ Tropical Medicine Institute "Pedro Kouri" (IPK), \\ Department of Epidemiology, Havana, Cuba, ${ }^{4}$ Tropical Medicine Institute "Pedro Kouri" (IPK), National Reference Laboratory for Pneumococcus, \\ Havana, Cuba \\ Keywords: cuba, streptococcus pneumoniae, burden of disease, pneumococcal conjugate vaccine \\ https://doi.org/10.29392/joghr.3.e2019071
}

\section{Journal of Global Health Reports}

Vol. 3, 2019

\section{Background}

The Cuban heptavalent conjugate pneumococcal vaccine will be introduced for children beginning in 2020. We estimated the burden of pneumococcal cases and deaths in children 1-59 months in 2015.

\begin{abstract}
Methods
Mortality and morbidity attributable to pneumococcus were estimated for each of the three primary syndromes commonly associated with pneumococcus: pneumonia, meningitis, and invasive non-pneumonia, non-meningitis (NPNM). Vaccine randomized clinical trial data were used to estimate the proportion of pneumonia deaths attributable to pneumococcus. Data from Cuba were obtained from two domestic sources: National Bacterial Meningitis Surveillance System and the laboratory register from the National Reference Laboratory. Syndrome-specific pneumococcal mortality proportions were applied to all-cause pneumonia and meningitis death estimates prepared by the World Health Organization Maternal Child Epidemiology Estimation (WHO/MCEE) collaboration. The proportion of pneumonia cases attributable to pneumococcus was applied to estimates of all-cause clinical and severe pneumonia also prepared by the WHO/MCEE collaboration. Pneumococcal NPNM morbidity and mortality estimates were prepared using the ratio pneumococcal meningitis to pneumococcal invasive disease. Estimates were adjusted for HIV prevalence and access to health care.
\end{abstract}

\section{Results}

In 2015, pneumococcus was estimated to cause 970 severe cases (uncertainty range, $\mathrm{UR}=692-1209)$ and 39 deaths (UR=23-59) among children 1-59 months. The estimated incidence rate of severe pneumonia was 149 (UR=112-170) per 100,000 children $1-59$ months. The estimated case fatality ratio (CFR) was $2 \%(\mathrm{UR}=1-2 \%)$. Over a period of one year, 22 deaths were attributed to pneumococcal pneumonia (UR=16-23), 9 (UR=14-19) to pneumococcal meningitis, and $8(\mathrm{UR}=3-17)$ to pneumococcal bacteremia. The CFR due pneumococcal meningitis was 18\% (UR= 8-37\%) in 2015.

\section{Conclusions}

Reduced morbidity and mortality due to pneumococcal disease in Cuba could be achieved with the accelerated introduction of a novel PCV product in children.

Streptococcus pneumoniae (pneumococcus) is a leading bacterial cause of pneumonia, meningitis, and other serious invasive diseases in children. In many settings, pneumococcal disease continues to represent an important cause of morbidity and mortality in children and the elderly. Researchers estimate that pneumococcus caused approximately 300,000 deaths in children globally in $2015 .^{1}$ The high burden of pneumococcal disease in many settings has been associated with substantial health care system costs to governments and society. 2,3
Recognizing the substantial burden of pneumococcal disease occurring in children and the established safety and efficacy of current pneumococcal conjugate vaccine (PCV) products available for use in this age group, the World Health Organization (WHO) recommends that all countries use PCV in their national routine immunization programs. ${ }^{4}$ Evidence from Latin America has demonstrated the significant impact of PCV on hospitalizations in children due to radiograph-confirmed pneumonia, clinical pneumonia, meningitis, and invasive pneumococcal disease. ${ }^{5}$ However, 
concerns about the cost PCV has been a barrier to its introduction in some countries. ${ }^{6}$

The Cuban government has a new heptavalent PCV product under advanced clinical development. ${ }^{7,8}$ The candidate vaccine includes contains $2 \mu \mathrm{g}$ of serotypes $1,5,14,18 \mathrm{C}$, $19 \mathrm{~F}, 23 \mathrm{~F}$ and $4 \mu \mathrm{g}$ of $6 \mathrm{~B}$ each conjugated to tetanus toxoid (TT). Pending positive clinical results, the Cuban government is planning to introduce the novel heptavalent PCV product in its national immunization program in 2019 using a $2+1$ schedule. ${ }^{9}$ In the meantime, a randomized clinical trial enrolling infants 2-6 months of age is being conducted to assess the efficacy of this vaccine for use in this age group (RPCEC00000243). Estimates of pneumococcal disease burden are needed to inform the use of this PCV product and to assess the potential impact of the new vaccine. We use globally established methods to estimate pneumococcal morbidity and mortality in children younger than 5 years in Cuba for 2015.

\section{METHODS}

We used the same methods previously published to estimate pneumococcal disease burden in Cuba. ${ }^{1}$ We updated a systematic review of pneumococcal invasive disease from 1980-2005 ${ }^{10}$ with published and unpublished data through 2014 for the meningitis and NPNM models. We followed the same methods and quality assessment criteria described previously. We searched six global databases (ie, PubMed, EMBASE, Biosis, Cochrane, Global Health, and Pascal) and five regional databases (ie, IMEMR, IMSEAR, LILACS, WHOLIS, WPRIM). Additional pneumococcal data were obtained from two domestic sources: National Bacterial Meningitis Surveillance System and the laboratory register from the National Reference Laboratory at Tropical Medicine Institute "Pedro Kouri". ${ }^{11}$ The mortality and morbidity attributable to pneumococcus in Cuba were estimated separately for pneumonia, meningitis, and NPNM. All analyses were conducted using Stata 14 of StataCorp (Stata Corp, College Station, TX, USA).

To estimate pneumococcal pneumonia mortality and morbidity, we applied etiologic fractions to estimates of allcause pneumonia deaths and cases in Cuba in 2015 prepared by WHO and the Maternal and Child Epidemiology Estimation (MCEE) collaboration ${ }^{12,13}$ For Cuba, all-cause pneumonia deaths are based on hospital-based vital registration data. Table 1 describes the ICD10 codes used to assign deaths to pneumonia and meningitis causes of death. ${ }^{14}$ All-cause pneumonia and severe pneumonia cases were based on the prevalence of pneumonia risk factors from Multiple Indicator Cluster Surveys conducted in Cuba. ${ }^{15-21}$ To determine the proportion of pneumonia mortality and morbidity attributable to pneumococcus, we used the vaccine probe approach we previously described in detail. 1,22 Briefly, efficacy values from PCV randomized control trials were adjusted to account for pneumococcal serotype distribution, incomplete vaccine efficacy, and the proportion of pneumonia attributable to Haemophilus influenzae type $b$ (Hib), since all PCV trials were conducted following the introduction of Hib vaccine. Following these adjustments, efficacy values were combined into summary estimates. We standardized WHO pneumonia case definitions. ${ }^{15,16,23}$ PCV trials did not assess vaccine efficacy against pneumonia mortality. Therefore, we used efficacy against radiographyconfirmed, primary end-point pneumonia as a proxy for this value.

We estimated pneumococcal meningitis deaths by applying an estimate of the proportion of meningitis deaths in Cuba attributable to pneumococcus to all-cause meningitis deaths in Cuba prepared by the WHO/MCEE collaboration. We divided the pneumococcal meningitis death estimate by an estimate of pneumococcal meningitis CFR for Cuba derived from the literature and adjusted for access to care. The proportion of meningitis deaths attributable to pneumococcus in Cuba was determined by meta-analyses of observational studies from the literature that provide data for the most common causes of bacterial meningitis (ie, pneumococcus, Hib, and Neisseria meningitidis). We estimated pneumococcal meningitis case fatality using data from the literature from Cuba and epidemiologically relevant settings. Reported case fatality values reflect mortality in children with access to care. Therefore, we adjusted estimates of pneumococcal meningitis case fatality to account for the higher case fatality assumed for those without access to care. We used the proportion of children seeking care for pneumonia symptoms from Multiple Indicator Cluster Surveys in Cuba as a proxy for this value.

We estimated pneumococcal morbidity due to other invasive disease using studies reporting on the ratio of pneumococcal NPNM cases to pneumococcal meningitis cases. Estimates from settings that are epidemiologically to Cuba in 2015 were then combined into summary estimates. We stratified pneumococcal NPNM cases by severe and non-severe cases. We used a summary estimate of case fatality for pneumococcal NPNM from the literature to estimate NPNM in children.

We estimated pneumococcal pneumonia and meningitis deaths in HIV-infected children using estimates of HIV prevalence in children less than 59 months provided by UNAIDS (personal communication) and estimates of the relative risk for invasive pneumococcal disease in HIV-infected children from published meta-analyses. ${ }^{22}$ Cuba has not yet introduced PCV and so we did not adjust for vaccine use for 2015. These estimates are reported with uncertainty ranges (UR) using the same methods described elsewhere. ${ }^{1}$

\section{RESULTS}

The estimates of pneumococcal disease burden for 2015 are presented in Table 2. We estimated that pneumococcus is responsible for 39 deaths in children 1-59 months in Cuba in 2015. 22 deaths were attributed to pneumococcal pneumonia (UR=16-23), 9 deaths to meningitis $(\mathrm{UR}=14-19)$ and 8 to bacteremia (UR=3-17). Despite the child mortality rate in children less than five years is low (5.5 per 1,000 live births) compared with other Latin America and the Caribbean countries, pneumococcal pneumonia deaths represent $14 \%$ of all deaths in children 1-59 months and pneumococcal meningitis deaths represent $5 \%$ of all deaths.

In addition to the 22 deaths ( $\mathrm{UR}=16-23)$ due to pneumococcal pneumonia in 2015, we estimated 1330 (UR=1149-1581) pneumococcal pneumonia cases, of which 862 (UR=64-983) were estimated to be severe pneumococcal 
Table 1. ICD codes for all-cause pneumonia and meningitis deaths in children 1-59 months (25)

\begin{tabular}{lll}
\hline Cause of death & ICD-10 codes & ICD-9 codes \\
\hline Acute respiratory infections & H65-H66, J00-J22, J85, P23, U04 & $460-466,480-487,381-382,513,770.0$ \\
Meningitis/encephalitis & A20.3, A32.1, A39.1, G00-G09 & $036,320,322-326$ \\
\hline
\end{tabular}

Table 2. Indicators used for modeling pneumococcal disease burden for Cuba in 2015

\begin{tabular}{ll}
\hline Values for Cuba in 2015 & Value \\
\hline Indicator and source & 587755 \\
Population less than 5 years in $2015(27)$ & 5.5 per 1000 live births \\
Child mortality rate in $2015(28)$ & $93 \%$ \\
Access to care in $2015(29,30)$ & \\
$\%$ of deaths in children 1-59 months due to meningitis in $2015(15)$ & \\
Pneumococcal conjugate vaccine coverage in 2015 (16) & $14 \%$ \\
\% of deaths in children 1-59 months due to pneumonia in 2015 (26) & \\
\hline
\end{tabular}

Table 3. Estimates of cases and deaths due to Pneumococcal pneumonia in Cuba in 2015

\begin{tabular}{lcc}
\hline & Estimate & Uncertainty range (UR) \\
\hline Pneumococcal pneumonia & & $198.52-273.50$ \\
\hline Incidence rate*, $\dagger$ & 1330 & $1147-1581$ \\
Cases $^{*}$ & 230.08 & $111.75-170.13$ \\
Incidence rate (severe cases) ${ }^{*} \dagger$ & 149.16 & $646-983$ \\
Severe cases* & 862 & $1-2 \%$ \\
CFR (clinical pneumonia) $†$ & $2 \%$ & $2.71-3.99$ \\
Mortality rate (HIV-uninfected) ${ }^{\dagger}$ & 3.83 & $16-23$ \\
Deaths: & 22 & $16-23$ \\
Deaths in HIV-uninfected children & 22 & $0-0$ \\
Deaths in HIV-infected children & 0 & \\
\hline
\end{tabular}

*HIV-infected and uninfected cases.

†Per 100,000 children 1-59 months.

¥HIV-uninfected deaths and cases.

pneumonia cases. Pneumococcal pneumonia deaths in Cuba correspond to a mortality rate of $4(\mathrm{UR}=3-4)$ deaths per 100,000 children 1-59 months. The pneumococcal pneumonia incidence rate in 2015 was 230 (UR=198-273) cases per 100,000 children and the severe pneumococcal pneumonia incidence rate was 149 (UR=112-170) cases per 100,000 children (Table 3)

Pneumococcal meningitis was estimated to cause 51 (UR=22-105) cases in 2015 with a corresponding incidence rate of $9(\mathrm{UR}=4-18)$ cases per 100,000 children 1-59 months. The pneumococcal meningitis case fatality in 2015 was estimated to be $18 \%$ (UR= 8-37\%) in 2015 (Table 4). Similarly, there were 152 ( $\mathrm{UR}=66-315)$ cases of non-severe pneumococcal NPNM in 2015 in Cuba and 57 (UR=25-119) cases of severe pneumococcal NPNM in 2015. The case fatality for pneumococcal NPNM was also high, at approximately $14 \%$ (UR=6-29\%) in 2015 (Table 5).

In summary in 2015 in Cuba, there were an estimated 970 (UR=692-1209) severe cases of pneumococcal disease and 39 deaths $(\mathrm{UR}=23-59)$ in children 1-59 months.

\section{DISCUSSION}

This paper documents the burden of pneumococcal disease Cuba using international standardized international methodology. ${ }^{1}$ The scope includes a description of invasive and non-invasive disease in children less than five years of age. This constitutes the first comprehensive country-level estimation of pneumococcal disease burden in Cuba that will allow policy maker to develop estimates of the potential impact of childhood disease intervention and determine the cost-effectiveness of vaccination.

Previous studies estimating the burden of pneumococcal meningitis in Cuban children have been conducted ${ }^{24-26}$ and were included as part of evidence revised to generate the WHO document reporting the Burden of Pneumococcal Disease and Cost-Effectiveness of a Pneumococcal Vaccine in Latin America and the Caribbean. ${ }^{27}$

Establishing the burden of disease and creating demand for the pneumococcal conjugate vaccine is one of the critical first steps required when considering the introduction of a new vaccine. ${ }^{28,29}$ Without recognition of the burden 
Table 4. Estimates of cases and deaths due to Pneumococcal meningitis in Cuba in 2015

\begin{tabular}{lcc}
\hline & Estimate & Uncertainty range (UR) \\
\hline Pneumococcal meningitis & & $3.77-18.25$ \\
Cases* $^{*}$ & 8.78 & $22-105$ \\
CFR $\neq$ & 51 & $8-37 \%$ \\
Mortality rate (HIV-uninfected) $\dagger$ & $18 \%$ & $0.68-3.28$ \\
Deaths: & 1.58 & $4-19$ \\
Deaths in HIV-uninfected children & 9 & $4-19$ \\
Deaths in HIV-infected children & 9 & $0-0$ \\
\hline
\end{tabular}

*HIV-infected and uninfected cases.

†Per 100,000 children 1-59 months.

$\ddagger$ HIV-uninfected deaths and cases.

Table 5. Estimates of cases and deaths due to Pneumococcal non-pneumonia, non-meningitis (NPNM) in Cuba in 2015

\begin{tabular}{lcc}
\hline & Estimate & Uncertainty range (UR) \\
\hline Pneumococcal non-pneumonia, non-meningitis (NPNM) & & $11.37-55.1$ \\
\hline Incidence rate (non-severe) ${ }^{*} \dagger$ & 26.5 & $66-318$ \\
Cases (non-severe) & 153 & $4.28-20.72$ \\
Incidence rate (severe) ${ }^{*} \dagger$ & 9.97 & $25-120$ \\
Cases (severe) & 58 & $6-29 \%$ \\
CFR (severe) $\ddagger$ & $14 \%$ & $0.6-2.92$ \\
Mortality rate (HIV-uninfected) $\dagger$ & 1,4 & $3-17$ \\
Deaths: & 8 & $3-17$ \\
Deaths in HIV-uninfected children & 8 & $0-0$ \\
Deaths in HIV-infected children & 0 & \\
\hline
\end{tabular}

CFR - case fatality rate

*HIV-infected and uninfected cases.

†Per 100,000 children 1-59 months.

$\ddagger$ HIV-uninfected deaths and cases.

of pneumococcal disease, health decision makers will underestimate the value of pneumococcal vaccination. However, estimating the total burden of pneumococcal disease is challenging because standard diagnostics have a low sensitivity and therefore, observational and surveillance studies systematically underestimate pneumococcal disease burden. In Cuba, the epidemiological surveillance system is currently being strengthened and will include collection of more epidemiological data including incidence data from sentinel sites.

The proportion of deaths in Cuban children 1-59 months due to pneumonia in 2015 (14\%) was lower than global estimation for the same year, ${ }^{1}$ but is 2 -fold greater than that reported for America region where several countries have already introduced pneumococcal vaccine as part of routine immunization programs ${ }^{30}$ According our currently estimations, the incidence rate of pneumonia in Cuban children, mortality rate and case fatality are very similar to global estimations for 2015. To establish population-based surveillance for X-ray confirmed pneumonia and data from some of these surveillance systems has recently been implemented in Cuba data including incidence data at three sen- tinel sites and a working group was created in each sentinel site to standardize the categorization of radiological pneumonia.

The median percentage of confirmed cases of bacterial meningitis due to pneumococcus reported in Cuba in 2002 was $27 \%$ (UR=26-28). For 2015, we reported 51 cases (UR=22 - 105) with higher incidence rate than reported for American region, but lower than the global estimation for the same year (13; UR=5-26). ${ }^{25}$ However, in comparison, the mortality is very similar than reported by Europe and American region. In addition, the case fatality is lower (18\%) compared with global estimations (44\%) and American countries (27\%).

The main limitation in our data about pneumococcal meningitis is associated with pneumococcal isolation in cerebral fluid and in general, the incidence of pneumococcus may have been underestimated due to lack of collection of blood cultures, poor yield of culture techniques, variation in laboratory practice (including hours of operation), prior antibiotic use and non-uniformly applied case definitions. In this way, the decision to implement a sentinel surveillance system four years prior to PCV introduction (ie, since 
2014) will substantially improve the data quality to conduct realistic estimation of burden disease that will permit international comparisons and to measure the impact of pneumococcal vaccine introduction.

\section{CONCLUSIONS}

Despite limitation in epidemiological surveillance and microbiological diagnostic that typically underestimate the true burden of pneumococcal disease, we can conclude that pneumococcal disease is relatively common in Cuba and represents an important public health problem. If we take into account that many of these infections are serious and may lead to hospitalizations, permanent disability, and death and the large burden of pneumococcal acute otitis media is also substantial contributor to healthcare system costs in Cuba, the introduction of a novel heptavalent PCV should be a high priority for the country as it will likely reduce the morbidity and mortality due to pneumococcal infections in Cuban children.

\section{ACKNOWLEDGEMENTS}

The authors thank the independent expert reviewers of the Global Burden of WHO Disease Spn Project from the Johns Hopkins Bloomberg School of Public Health, specifically Katherine L O'Brien and Maria Knoll, who advised in the conceptual and methodology strategy. We also thank for Department of Epidemiology and National Reference Laboratory of Pneumococcus from the Tropical Medicine Institute "Pedro Kourí" (IPK) and the Cuban National Immunization Programs of Ministers of Public Health of Cuba. The authors also want to thank the professional members of the Cuban Pneumococcal Clinical Research, Surveillance and Impact Evaluation Working Group: Isabel Luis Gonzálvez, Anais García-Fariñas, Meiby C. Rodríguez, Beatriz Paredes, Mayelín Mirabal, Ivan Cuevas, Marlene Armesto, Raúl Mujica, Anais Linen, Dagmar García-Rivera, Laura M. Rodriguez, Amarilis Pérez Baños; Rocmira Pérez Nicado, Aniurka Garcés Hechavarría, Dayana L. Castellanos Almaguer, Yamilka Soroa Millán y Dayle Martínez Bedoya, Darielys Santana, Marisel Martínez, Darielys Santana, Marisel Martínez, Ubel Ramirez, Jessy Pedroso, Felix Cardoso, Annette Villar y Humberto González, Yuri Valdés-Balbín and Vicente Verez-Bencomo from Finlay Vaccine Institute; Susana Borroto from Tropical Medicine Institute "Pedro Kourí; Martha Marrero, Ana María Gálvez from Public Health National School; and the Clinical Research, Surveillance and Impact Evaluation Sentinel Network of Pneumococcal Project: Maria F Casanova, Dunia Chavez, Alaín Reyes, Mercedes Fonseca, Martha Montes de Oca, Nancy Nerelis Rodríguez Valladares, Antonio Velázquez Águila, Iliana García Rodríguez, Cary Tere Llul Tombo and Juan C. Yanes Macías (Cienfuegos); Maria Elena Mesa and Yariser Ricardo, Niurka Molina Águila, Ernesto Legarreta Peňa, Dania Vega
Mendoza, Gladys Abreu Gladys Fuentes, Miosotis Pérez Orta, Concepción Infante, María Cristina Reyes López, Lidia Teresita Ramos (Havana); Josefina Herrera López, Nordis Caridad Donatién Rojas, Lissett Batista Caluff, Nivia Cobas Limonta, Moraima, Silvia Justiz Hernández, Malfran García, Luis Enrique Rondón Rondón, Bertha Isabel Rondón Peña, Magdevis Pajaro Medina, Daisy Díaz Teran, Mariela Cuza Ferrer, María Maren González, Samira Maceira Soto, Itciar Áreas, José R. Guillén and Luis Váldez (Santiago de Cuba).

\section{ETHICS APPROVAL}

This study was submitted to the Tropical Medicine Institute "Pedro Kourí" Institutional Ethics Committee (IPK-IEC) and was approved under the agreement IPK IEC \# 17-14/37-16. In addition, the Scientific Council of the Finlay Vaccine Institute approved the study.

\section{AVAILABILITY OF DATA AND MATERIALS}

The data are available from the corresponding author (Nivaldo Linares-Pérez, (nlinares@finlay.edu.cu) upon reasonable request, subject to obtaining clearance from the Pneumococcal Project Coordination Working Group of the Finlay Vaccine Institute of Cuba. For information on how to obtain clearance to access the data, contact the Pneumococcal Project Coordinator at +5372086086 or dsantana@finaly.edu.cu.

\section{FUNDING}

This study is made possible by the generous support of the Cuban people through the Funds Fund for Research, Science and Innovation of the Ministry of Science, Technology and Environment, under the terms of the Contracts Project $0501195-25 / 2017$. The contents are the responsibility of the authors and do not necessarily reflect the views of Governmental Funds Fund for Research, Science and Innovation of the Ministry of Science, Technology and Environment or the Cuban Government.

\section{COMPETING INTERESTS}

The authors completed the Unified Competing Interest form at http://www.icmje.org/coi_disclosure.pdf (available upon request from the corresponding author), and declare no conflicts of interest.

\section{CORRESPONDENCE TO:}

Nivaldo Linares-Pérez

Finlay Vaccine Institute

Av. 21 \#19810 e/198 y 200. Atabey. Playa.

P.O. Box 16042

Havana

Cuba CP: 11600

nlinares@finlay.edu.cu 


\section{REFERENCES}

1. Wahl B, O’Brien KL, Greenbaum A, et al. Burden of Streptococcus pneumoniae and Haemophilus influenzae type $\mathrm{b}$ disease in children in the era of conjugate vaccines: global, regional, and national estimates for 2000-15. Lancet Glob Health. 2018;6(7):e744-e757. doi:10.1016/s2214-109x(18)302 $\underline{47-\mathrm{x}}$

2. Prato R, Tafuri S, Fortunato F, Martinelli D. Why it is still important that countries know the burden of pneumococcal disease. Hum Vaccin. 2010;6(11):918-921. doi:10.4161/hv.6.11.13352

3. World Health Organization. Pneumococcal conjugate vaccine for childhood immunization-WHO position paper. Wkly Epidemiol Rec. 2007;23:93-104.

4. World Health Organization. Pneumococcal vaccines: WHO position paper-2012. Weekly Epidemiological Record=Relevé épidémiologique hebdomadaire. 2012;87:129-144.

5. de Oliveira LH, Camacho LAB, Coutinho ESF, et al. Impact and effectiveness of 10 and 13-valent pneumococcal conjugate vaccines on hospitalization and mortality in children aged less than 5 years in Latin American Countries: A systematic review. PLoS One. 2016;11(12):e0166736. doi:10.1371/journal.pon e.0166736

6. Saokaew S, Rayanakorn A, Wu DBC, Chaiyakunapruk N. Cost effectiveness of pneumococcal vaccination in children in low- and middle-income countries: A Systematic review. Pharmacoeconomics. 2016;34(12):1211-1225. doi:10.1 007/s40273-016-0439-3

7. Martínez CPD, Linares-Pérez N, Toledo-Romaní ME, et al. Safety and immunogenicity of the Cuban heptavalent pneumococcal conjugate vaccine in healthy infants. Results from a double-blind randomized control trial Phase I. Vaccine. 2018;36(32):4944-4951. doi:10.1016/j.vaccine.2018.0 $\underline{5.027}$

8. Dotres CP, Puga R, Ricardo Y, et al. Safety and preliminary immunogenicity of Cuban pneumococcal conjugate vaccine candidate in healthy children: a randomized phase I clinical trial. Vaccine. 2014;32(41):5266-5270. doi:10.1016/i.vaccine.2014.0 $\underline{6.094}$

9. Linares-Pérez N, Toledo-Romaní ME, SantanaMederos D, et al. From individual to herd protection with pneumococcal vaccines: the contribution of the Cuban pneumococcal conjugate vaccine implementation strategy. Int J Infect Dis. 2017;60:98-102. doi:10.1016/j.ijid.2017.03.011
10. Deloria Knoll M, O’Brien K, Henkle E, et al. Global Literature Review of Haemophilus Influenzae Type $b$ and Streptococcus Pneumoniae Invasive Disease among Children Less than Five Years of Age, 1980-2005. World Health Organization; 2009.

11. Toraño-Peraza G, Pías-Solis L, Abreu-Capote M, Rodríguez-Ortega M, Dickinson-Meneses F, Varcárcel-Sánchez M. Serotipos y resistencia antimicrobiana de aislamientos meníngeos de Streptococcus pneumoniae. Cuba, 2007-2012. Vaccimonitor. 2014;23:117-123.

12. Liu L, Oza S, Hogan D, et al. Global, regional, and national causes of under-5 mortality in 2000-15: an updated systematic analysis with implications for the Sustainable Development Goals. Lancet. 2016;388(10063):3027-3035. doi:10.1016/s0140-673 $\underline{6(16) 31593-8}$

13. McAllister DA, Liu L, Shi T, et al. Global, regional, and national estimates of pneumonia morbidity and mortality in children younger than 5 years between 2000 and 2015: a systematic analysis. Lancet Glob Health. 2019;7:e47-e57. doi:10.1016/s2214-109x(18)3 0408-x

14. World Health Organization. MCEE-WHO methods and data sources for child causes of death 2000-2015. Global Health Estimates Technical Paper WHO/HIS/ IER/GHE/2016.1. Accessed July 18, 2017. http://ww w.who.int/healthinfo/global_burden_disease/ChildCO D_method_2000_2015.pdf

15. World Health Organization. Programme for the Control of Acute Respiratory Infections: Technical Bases for the WHO Recommendations on the Management of Pneumonia in Children at First Level Health Facilities. World Health Organization; 1991.

16. World Health Organization. Handbook IMCI: Integrated Management of Childhood Illness. World Health Organization; 2005.

17. Johnson HL, Deloria-Knoll M, Levine OS, et al. Systematic evaluation of serotypes causing invasive pneumococcal disease among children under five: the pneumococcal global serotype project. PLoS Med. 2010;7(10):e1000348. doi:10.1371/journal.pmed.1000 $\underline{348}$

18. United Nations Department of Economic and Social Affairs Population Division. World Population Prospects: The 2015 Revision. DVD Edition. United Nations; 2015. 
19. United Nations Interagency Group for Child Mortality Estimation (IGME). The 2015 Revision. United Nations; 2015.

20. Hirai M, Grover N, Huang C. The Measurement of Non-Communicable Diseases in 25 Countries with Demographic and Health Surveys. DHS Occasional Papers No. 10. ICF International. Published 2015. Accessed June 30, 2018. http://dhsprogram.com/data/

21. UNICEF. Monitoring the situation of children and women. Multiple Indicator Cluster Surveys (MICS). Published 2015. Accessed June 30, 2018. http://mics.u nicef.org/

22. O’Brien KL, Wolfson LJ, Watt JP, et al. Burden of disease caused by Streptococcus pneumoniae in children younger than 5 years: global estimates. Lancet. 2009;374(9693):893-902. doi:10.1016/s0140-6 736(09)61204-6

23. Cherian T, Mulholland EK, Carlin JB, Ostensen H, Amin R, de Campo M. Standardized interpretation of paediatric chest radiographs for the diagnosis of pneumonia in epidemiological studies. Bull World Health Organ. 2005;83:353-359.

24. Pérez AE, Dickinson FO, Rodríguez M. Community acquired bacterial meningitis in Cuba: a follow up of a decade. BMC Infect Dis. 2010;10:130-136. doi:10.118 6/1471-2334-10-130
25. Dickinson Meneses FO, Pérez Rodríguez AE. Las meningoencefalitis bacterianas en la población infantil cubana: 1998-2000. Rev Cubana Pediatr. 2002;74:106-114.

26. Dickinson Meneses FO, Pérez Rodríguez AE. Meningoencefalitis bacterianas en Cuba. Rev Cuba Hig Epidemiol. 2001;39:86-94.

27. Constenla D, Gomez E, de la Hoz FP, O’Loughlim R, Sinha A, Valencia JE. The Burden of Pneumococcal Disease and Cost-Effectiveness of a Pneumococcal Vaccine in Latin America and the Caribbean. Sabin Vaccine Institute; 2007.

28. Burchett HED, Mounier-Jack S, Griffiths UK, Mills AJ. National decision-making on adopting new vaccines: a systematic review. Health Policy Plan. 2012;27(Suppl 2):ii62-ii76. doi:10.1093/heapol/czr049

29. Burchett HED, Mounier-Jack S, Griffiths UK, et al. New vaccine adoption: qualitative study of national decision-making processes in seven low- and middleincome countries. Health Policy Plan. 2012;27(suppl 2):ii5-ii16. doi:10.1093/heapol/czs035

30. International Vaccine Access Center (IVAC). VIEW-hub. Accessed June 27, 2018. http://www.viewhub.org 